\title{
REVISIONES
}

\section{Cambio representacional en los padres: reconstrucción de teorías subjetivas de la prevención del consumo de drogas en los hijos e hijas*}

\author{
Representational change in parents: reconstruction of subjective theories \\ of drug prevention in sons and daughters
}

\author{
Transformação representacional em pais: reconstrução de teorias \\ subjetivas da prevenção do consumo de drogas nos filhos e filhas
}

\section{David Cuadra Martínez}

Universidad Santo Tomás, Sede Copiapó, 84332846, cuadradavid2@gmail.com

\begin{abstract}
RESUMEN
Se describe el cambio representacional producido por una taller educativo de reconstrucción de teorías subjetivas del consumo de drogas y su prevención, en 16 padres de una caleta de pescadores, de la región de Atacama, Chile. Se aplicaron tres grupos de discusión y los datos obtenidos se procesaron mediante la técnica de análisis de contenido de la grounded theory. El cambio se produjo en las teorías subjetivas epistemológicas, que enjuician el conocimiento, enseñanza y aprendizaje de la prevención y específicas de consumo de drogas y su prevención, manifestándose en el desarrollo de una mayor complejidad de sus explicaciones y agentividad en la crianza preventiva. Éste se facilita por la explicitación sucesiva de las teorías subjetivas, reflexión, trabajo grupal, relación de confianza aprendiz-educador, uso de lenguaje con sentido y presentación de información científica del tema. En la discusión se analiza este cambio representacional y su implicancia en la educación parental.
\end{abstract}

Palabras clave: cambio representacional, teorías subjetivas, padres, prevención, consumo de drogas.

\section{ABSTRACT}

This paper describes the representational change produced by an educational workshop of subjective theories reconstruction of drug abuse and its prevention in 16 parents of a fishing cove in Región de Atacama, Chile. Three discussion groups were applied, and the data obtained were processed using the technique of content analysis of the grounded theory. The change was observed in the subjective epistemological theories, which deal with knowledge, teaching and learning of prevention, and specific of drug abuse and its prevention; all demonstrated by the development of more complex explanations and an agentivity in preventive parenting. This is facilitated by the successive explanation of subjective theories, reflection, group-work, trusting relationship learner-educator, sense language use and presentation of scientific information on the topic. In discussion, this representational change and its implication on parental education is analyzed.

Keywords: representational change, subjective theories, parents, prevention, drug use.

\section{RESUMO}

Descreve-se a transformação representacional produzida por uma oficina educativa de reconstrução de teorias subjetivas ao consumo e prevenção de drogas em 16 pais de uma aldeia de pescadores, na região do Atacama, Chile. Foram realizados três grupos de discussão e os dados obtidos foram processados utilizando-se a técnica de análise de conteúdo da Teoria Fundamentada nos Dados. As transformações ocorreram nas teorias subjetivas

Este estudio es fruto de un proyecto financiado por el Gobierno Regional de Atacama, a través del Concurso de Actividades de Carácter Social y Rehabilitación de Drogas, Glosa Ley 20.641, Región de Atacama, Chile. 
Estudios Pedagógicos XLII, Nº 1: 283-298, 2016

CAMBIO REPRESENTACIONAL EN LOS PADRES: RECONSTRUCCIÓN DE TEORÍAS SUBJETIVAS DE LA

PREVENCIÓN DEL CONSUMO DE DROGAS EN LOS HIJOS E HIJAS

epistemológicas que julgam o conhecimento, ensino e aprendizagem da prevenção e específicas relacionadas ao consumo e prevenção de drogas, manifestando-se no desenvolvimento de uma maior complexidade de suas explicações e agentividade na criança preventiva. Isto é facilitado pela explicitação sucessiva de teorias subjetivas, reflexão, trabalho em grupo, relação de confiança aluno-professor, o uso da linguagem com sentido e apresentação de informações científicas sobre o assunto. Na discussão, analisa-se esta transformação representacional e sua implicação na educação dos pais.

Palavras-chave: transformação representacional, teorias subjetivas, pais, prevenção, consumo de drogas.

\section{INTRODUCCIÓN}

Las investigaciones sobre el consumo de drogas y su prevención han presentado evidencias de la importancia de la familia como factor de riesgo o de protección (Alfonso, Huedo-Medina y Espada, 2009; Larriba, Durán y Suelves; 2006; Vielva, 2001), y han señalado que las creencias que tienen de las drogas son un factor importante en las conductas de consumo en los hijos(as) (Becoña, 2001; Pons y Berjano, 1999; Secades, Fernández y Vallejo, 2005).

Nuño y González (2004) encontraron que algunos padres presentan creencias irracionales sobre el consumo de drogas en hijos(as): básicamente, de invulnerabilidad frente a este problema, sobreresponsabilidad personal cuando se rehabilita y una desajustada esperanza en la ayuda que proporcionan los centros de tratamiento, lo que se asociaría a una disfuncionalidad en la crianza. Otros estudios se han focalizado en las explicaciones que los padres dan a sus hijos(as) del consumo de drogas, encontrando que cuando son insuficientes y contradictorias, tenderían a buscar información en otras fuentes de conocimiento (Sierra et al. 2005), mientras que cuando se presentan explicaciones con argumentos objetivos y adecuados, tendrían una menor posibilidad de llegar a ser consumidores (Navarro, 2001; Secades, 1996).

Las explicaciones que las personas tienen constituyen un tipo particular de creencias que regulan de manera importante el comportamiento. Han sido denominadas teorías subjetivas (Catalán, 2010; Flick, 2004; Grotjahn, 1991), implícitas (Oliva, 1999; Pozo, 1999; Rodrigo, Rodríguez y Marrero, 1993) o de dominio (Carey, 1985), por su estructura argumentativa, su similitud con las teorías científicas y su mayor generalización. Las teorías subjetivas se diferencian porque se sustentan en supuestos ontológicos y epistemológicos que otorgan mayor valor a la racionalidad y proactividad del ser (Catalán, 2010), mientras que las teorías implícitas o de dominio se caracterizan por un mayor grado de inconsciencia en la persona (Pozo, 2002). Así, desde esta corriente teórica, es posible decir que las teorías subjetivas que los padres presentan sobre el consumo de drogas y su prevención, se encuentran relacionadas con las pautas de crianza que aplican con sus hijos(as) para abordar este problema y, por lo tanto, que las intervenciones orientadas al cambio de esta representación subjetiva, se asociarían a una mayor funcionalidad en la crianza preventiva.

El cambio subjetivo en contextos educativos ha sido considerado como un cambio representacional (Marín, 1999; Oliva, 1999; Pozo, 1999), conceptual (Driver et al. 1994), concepcional (Moreira y Greca, 2003) o fuerte (Carey, 1985). Básicamente, las ideas previas o preconcepciones que presentan los aprendices influyen de manera importante en el aprendizaje que la escuela pretende desarrollar. Éstas se encuentran organizadas en un sistema supraordenado de teorías que muchas veces no son conscientes, subordinan a 
otras creencias y son resistentes al cambio, lo que dificulta el aprendizaje del conocimiento formal o científico. Desde este enfoque, el cambio consiste en una nueva forma de concebir las cosas y se produce por: (a) la explicitación sucesiva de estas representaciones; (b) el uso de códigos lingüísticos comprensibles para los aprendices; (c) el uso de contenidos científicos o escolares como medio para desarrollar representaciones más elaboradas y (d) una enseñanza como proceso de contrastación y argumentación de modelos de conocimiento (Pozo, 1999).

Silveira (1991) propone utilizar inicialmente las preconcepciones de los estudiantes para explicar los fenómenos y, desde éstas, generar un conflicto cognitivo que permita cuestionarlas, lo que es similar a lo propuesto por Carey (1985), que señala que el cambio se produciría cuando las preconcepciones no logran explicar los fenómenos. Pozo y Gómez (1998) han considerado que éste se produce más bien desde un nivel ontológico y epistemológico, y consistiría en un enriquecimiento de las teorías personales.

El cambio de las teorías subjetivas ha sido menos estudiado y los principales aportes provienen de la psicoterapia (Castro, 2008; Castro y Cárcamo, 2012). Krause y Dagnino (2006) describen un proceso de cambio subjetivo complejo, que ocurre en varias etapas y culmina con la elaboración de una nueva teoría subjetiva acerca de sí mismo y el mundo, siendo los indicadores genéricos de cambio: (a) la aceptación del terapeuta como profesional competente, (b) la expresión de esperanza y necesidad de cambio, (c) reconstrucción de constructos subjetivos de sí mismo, referidos a la necesidad de cambio y la propia capacidad, y (d) construcción de una nueva teoría subjetiva, entre otros. Esta reconstrucción se produciría por la incorporación de nuevos significados, a través de la interacción con el terapeuta, siendo posibles los siguientes resultados: (a) aceptación e integración del nuevo contenido; (b) aceptación insuficiente del nuevo contenido; (c) no aceptación ni integración o incorporación rígida.

Por otra parte, Castro (2008) y Castro y Cárcamo (2012) describen el proceso de cambio en las teorías subjetivas, desde un contexto de educación formal de tipo continua. Específicamente, cómo un grupo de profesores cambia sus teorías subjetivas respecto la enseñanza y aprendizaje de valores, a través de una instancia de capacitación en la temática. Los principales cambios se producen por la reflexión colectiva sistemática sobre su propia práctica, lo que promueve el desarrollo de teorías subjetivas más complejas, que otorgan mayor control en este proceso y agentividad.

En la prevención familiar del consumo de drogas, el objetivo de los programas ha sido principalmente informar sobre el tema (Lloret et al. 2013), obteniendo bajos resultados (Rementería, 2001). Se asume que la sola exposición de conocimiento científico sobre drogas producirá el aprendizaje de la prevención, dejando de lado la subjetividad o, desde Tsukame (2002), el sentido subjetivo que los grupos le atribuyen.

Dicho lo anterior, los resultados que aquí se exponen corresponden a una investigación cualitativa que buscó dar respuesta a las siguientes interrogantes: ¿Qué cambio representacional se produce en los padres, al participar de un programa preventivo de reconstrucción de teorías subjetivas sobre consumo de drogas y su prevención? ¿cómo se produce el cambio de estas teorías subjetivas? y ¿qué factores promueven el proceso de reconstrucción?

El objetivo general fue describir el proceso de cambio representacional, producido por una taller educativo de reconstrucción de teorías subjetivas del consumo de drogas y su prevención, en padres de una caleta de pescadores de la región de Atacama, Chile. 
A nivel específico se buscó (a) describir los principales procesos de cambio de estas teorías subjetivas, (b) determinar los elementos del programa que se asocian a este proceso reconstructivo, y (c) proponer un modelo comprensivo del proceso de cambio representacional, desde la reconstrucción de estas teorías subjetivas, que contribuya a la prevención familiar de consumo de drogas.

Esta investigación cobra pertinencia y relevancia, dado que el consumo de drogas se considera actualmente un problema social en aumento, para el que se requieren soluciones urgentes (Elzo et al. 2000). Desde estos hallazgos, es posible comprender la implicancia que tienen las teorías subjetivas en la crianza preventiva del consumo de drogas en los hijos(as) y cómo reconstruirlas, a fin de promover una mayor funcionalidad de este tipo de pensamiento, constituyéndose en un aporte para la teoría de cambio representacional y, principalmente, para la educación preventiva familiar, dada la importancia que tiene este grupo como factor protector o de riesgo (Martínez, 2001; Pons y Berjano, 1999; Vielva, 2001), todo ello respondiendo a la necesidad de presentar evidencias de los resultados obtenidos en este tipo de intervenciones (Brotherhood y Sumnall, 2011; Lloret et al. 2013; Saez, 2002).

\section{MÉTODO}

\subsection{TIPO DE ESTUDIO, METODOLOGÍA Y DISEÑO}

Este estudio corresponde a uno de tipo descriptivo interpretativo, que utilizó una metodología y un diseño de evaluación cualitativos, dado que se buscó comprender el problema desde el propio marco interpretativo de los participantes, de manera inductiva, naturalista, holista y humanista (Taylor y Bogdan, 1987).

Respecto al diseño, la evaluación cualitativa está dirigida a evaluar programas mediante un contacto intenso con los sujetos, poniendo como eje la interpretación que hacen los propios actores de un proyecto, programa o intervención (Burgos, 2011). El objeto de evaluación fue un taller educativo dirigido a 16 padres y madres, que implementé con el objeto de generar un cambio en las teorías subjetivas sobre prevención del consumo de drogas en los hijos(as).

El taller educativo se sustentó en la teórica del cambio representacional (Pozo, 1999), específicamente, de teorías subjetivas (Castro, 2008; Castro y Cárcamo, 2012; Catalán, 2010; Flick, 2004; Krause y Dagnino, 2006), y tuvo una duración de 13 sesiones de una hora de trabajo, distribuidas en 6 meses y con los siguientes módulos:

en el primero, en 2 sesiones se aplicaron 2 grupos de discusión a fin de identificar, describir e interpretar las teorías subjetivas epistemológicas y específicas del consumo de drogas y su prevención;

en el segundo, se realizaron 2 sesiones, con el objetivo de que los participantes comprendieran la relación entre las teorías subjetivas y la acción, utilizando ejemplos y ejercicios vinculados a la vida cotidiana y laboral de los participantes;

en el tercero, constituido por 3 sesiones, se buscó el autoconocimiento y juicio crítico de las teorías subjetivas epistemológicas encontradas, a fin de que analizaran su disposición al aprendizaje y la posibilidad de cambio a partir de esta forma de pensar;

en el cuarto, comprendido por 3 sesiones, se buscó el autoconocimiento y juicio crítico de las teorías subjetivas que explican lo que es consumo de drogas y su prevención, incluyendo 
como herramientas de cambio (1) la presentación textual de las teorías subjetivas encontradas (power point y guía de trabajo) en un primer nivel de interpretación, representado por citas textuales de los participantes, y un segundo nivel de interpretación, constituido por la teoría subjetiva elaborada en palabras de los investigadores, (2) el análisis grupal de la orientación a la acción derivada de las teorías subjetivas y su implicancia en las pautas de crianza preventiva del consumo de drogas, y (3) ejercicios de enjuiciamiento del argumento de las teorías subjetivas encontradas, principalmente de la fuente de conocimiento, las evidencias propuestas y la utilidad de esta forma de pensar;

el quinto módulo consistió en la realización de 2 sesiones de análisis grupal de información científica sobre prevención familiar del consumo de drogas, contenida en una guía educativa; en el sexto, en 1 sesión se aplicó 1 grupo de discusión a fin de identificar el cambio de teorías subjetivas y los factores que lo promovieron.

\subsection{PARTICIPANTES}

Los participantes corresponden a 16 padres y madres, socios de una organización de pescadores artesanales de una caleta de la región de Atacama, Chile, de nivel socioeconómico bajo, con bajo nivel de escolaridad y cuyo oficio consiste en la extracción artesanal de recursos marinos. Se utilizó un muestreo por conveniencia, dado que este grupo fue beneficiario del taller educativo preventivo del consumo de drogas en hijos(as). La localidad de residencia se caracteriza por presentar indicadores altos de vulnerabilidad social.

Tabla 1. Caracterización de los participantes

\begin{tabular}{|c|c|c|c|c|c|c|c|c|}
\hline \multicolumn{2}{|c|}{$\begin{array}{c}\text { Rango de edad } \\
\text { (años) }\end{array}$} & \multicolumn{3}{c|}{$\begin{array}{c}\text { Escolaridad } \\
(\%)\end{array}$} & \multicolumn{2}{c|}{ Sexo } & $\begin{array}{c}\text { Nivel } \\
\text { Socioeconómico }\end{array}$ \\
\hline $31-40$ & $41-50$ & 50 o más & Básica & Media & $\begin{array}{c}\text { Superior } \\
\text { incompleta }\end{array}$ & Hombre & Mujer & Bajo \\
\hline $18 \%$ & $25 \%$ & $57 \%$ & $32 \%$ & $62 \%$ & $6 \%$ & $94 \%$ & $6 \%$ & $100 \%$ \\
\hline
\end{tabular}

\subsection{PROCEDIMIENTO DE RECOLECCIÓN DE INFORMACIÓN}

Se contactó a los padres del sindicato de trabajadores de pescadores y se les explicó los fines de la investigación, los criterios éticos y solicitó colaboración para participar del estudio. Los datos se recolectaron en el lugar de residencia de los padres, específicamente en la sede del sindicato, en dos momentos: antes y después de la intervención.

Se aplicaron tres grupos de discusión, dos antes de la intervención y uno al finalizarla, cada uno con una duración aproximada de 1 hora. Los grupos de discusión aplicados antes de la intervención estuvieron constituidos por los 16 padres beneficiarios del taller preventivo, distribuyéndose en 8 padres por cada uno. Se basaron en un guión temático que consideró los siguientes ejes: teorías subjetivas acerca del conocimiento, enseñanza y aprendizaje de la prevención del consumo de drogas; teorías subjetivas acerca del consumo de drogas y teorías subjetivas acerca de la prevención del consumo de drogas en los hijos(as).

El grupo de discusión aplicado al finalizar la intervención quedó constituido por 10 padres, seleccionados bajo el criterio de representatividad de casos, considerando distintos 
rangos de edad, género y nivel de escolaridad. Además, incorporó como criterio de inclusión el haber tenido al menos el $85 \%$ de asistencia al taller educativo. Esta última aplicación se apoyó en el guión temático ya indicado, agregando dos tópicos nuevos: procesos de cambio logrados a través del taller y factores que facilitaron el cambio.

La aplicación de los grupos de discusión se basó en la propuesta de Flick (2004), de indagación de episodios biográficos, por ser facilitadores de la producción de teorías subjetivas. Así, se consideró la exploración de episodios específicos informados espontáneamente, episodios repetidos informados espontáneamente y definiciones subjetivas de los tópicos abordados. Además, incluyó la propuesta de Castro (2008), de indagación de episodios tipo, que permiten respuestas hipotéticas de lo que haría el participante frente a un episodio ficticio propuesto por el investigador. Los datos obtenidos se grabaron en audio y posteriormente fueron transcritos.

\subsection{PROCEDIMIENTO DE ANÁLISIS DE LOS DATOS}

Los textos obtenidos de la transcripción de los grupos de discusión se procesaron mediante la técnica de análisis de contenido propuesta por la grounded theory (Strauss y Corbin, 2002). El análisis de los datos obtenidos antes de la intervención permitió describir e interpretar las teorías subjetivas epistemológicas y específicas de consumo de drogas y su prevención, además de constituir el material de trabajo durante la intervención. Mientras que el análisis de los datos del último grupo de discusión permitió identificar los principales cambios en las teorías subjetivas y los factores asociados al mismo, a partir del relato de los participantes y la contrastación con el contenido y estructura de las teorías subjetivas iniciales. Se aplicaron tres tipos de codificación:

Codificación abierta: Mediante el método comparativo constante, el texto transcrito se transformó en códigos más generales representados por teorías subjetivas identificadas a partir de su estructura argumentativa, compuesta por enunciados del tipo si entonces, porque, esto conlleva a aquello, y de teorías implícitas o deducidas por el propio investigador a partir del sentido explicativo extraído del texto (Catalán, 2010; Flick, 2004).

Codificación axial: Los códigos obtenidos se contrastaron mediante el método comparativo constante a fin de construir categorías que agruparon a un conjunto de teorías subjetivas. Las familias semánticas resultantes fueron: (a) reconstrucción de teorías subjetivas epistemológicas, que describe los cambios ocurridos en las explicaciones de los participantes acerca del conocimiento, la enseñanza y el aprendizaje de la prevención del consumo de drogas; (b) reconstrucción de las teorías subjetivas del consumo de drogas, que describe los cambios ocurridos en las explicaciones del consumo de drogas; (c) reconstrucción de las teorías subjetivas sobre prevención del consumo de drogas en los hijos(as), que describe los cambios ocurridos en las explicaciones de la prevención del consumo de drogas en los hijos(as); y (d) el motor del proceso de reconstrucción de las teorías subjetivas, que considera seis subcategorías que describen los elementos favorecedores del cambio.

Codificación selectiva: Se establecieron relaciones entre categorías a fin de elaborar una teoría comprensiva del fenómeno estudiado. La categoría núcleo en torno a la cual se agruparon las familias semánticas correspondió a la teoría subjetiva reconstruida. Además, se asumió la propuesta de Catalán (2010) respecto el análisis de contenido específico para teorías subjetivas, considerando la orientación a la acción de las mismas, que estuvo representada por las pautas de crianza y su estructura. 


\section{RESULTADOS}

\subsection{RECONSTRUCCIÓN DE LAS TEORÍAS SUBJETIVAS EPISTEMOLÓGICAS}

El principal proceso de cambio de las teorías subjetivas del conocimiento, enseñanza y aprendizaje de la prevención, implicó el otorgamiento de una mayor importancia al conocimiento y aprendizaje del tema, lo que se podría asociar a una mejor disposición a participar de este tipo de talleres educativos. Así lo expresa uno de los participantes:

Tener un poquito más de conocimiento, así pa poder saber a qué atenerse, porque... si te toca alguna vez... (p4, gdI). ${ }^{1}$

Yo voy igual... si yo tuviera más hijos chicos igual trataría de prevenir el consumo de drogas en ellos. Entonces eso lo aprendí acá, porque antes yo no pensaba así (p4, gdIII).

Los participantes también logran desarrollar una explicación de mayor complejidad de la enseñanza y el aprendizaje de la prevención, avanzando desde un planteamiento limitado, que condiciona la posibilidad de enseñar y aprender del tema, a la experiencia personal de consumo de drogas de quien educa, a uno más amplio, que considera diversas formas de enseñanza y aprendizaje de la prevención. Implica un proceso reconstructivo que le otorga mayor adaptación, dado que podría orientar su acción a aprender con mayor disposición, independiente del tipo de método de enseñanza utilizado.

Que no es necesario que él haya consumido para que nos enseñe a ver las cosas de la situación a como estamos hoy en día. Yo me di cuenta... me di cuenta que no era necesario... porque la mente... sentirlo como sí pu, él consume para que él nos enseñe. Erróneo, erróneo (p2, gdIII). ...no es necesario consumir para poder hablar de prevención... son creencias no más, se cree que porque yo consumo yo puedo hablar del tema pero si yo no consumo no puedo hablar del tema... (p3, gdIII).

\subsection{RECONSTRUCCIÓN DE LAS TEORÍAS SUBJETIVAS DEL CONSUMO DE DROGAS}

Inicialmente, el consumo de drogas es explicado apelando a factores personales y sociales, tales como las características de personalidad del consumidor y la presencia de un contexto comunitario que lo favorece. El cambio de la teoría subjetiva implica una mayor elaboración de la explicación, incorporando al grupo familiar como el factor más importante, situación que podría asociarse a una mayor agentividad en la prevención del consumo de drogas en los hijos(as).

Yo creo que lo más cercano, lo más cercano que lo lleva a uno a consumir, a uno cuando joven, el ambiente, no como uno los crie, porque uno cría y no sabe lo que cría en el fondo... (p9, gdII). Para mí la familia es el pilar número uno para educar y hacer todo lo humano posible para la familia, ahí parte todo, si está mal la familia está todo mal... (p6, gdIII).

La reconstrucción de la teoría subjetiva también considera una mayor atribución de consecuencias negativas al consumo de cualquier tipo de drogas y un impacto más

$\mathrm{P}=$ participante $; \mathrm{gdI}$ y gdII = grupos de discusión realizados antes de la intervención; gdIII = grupo de discusión realizado al finalizar la intervención. 
sistémico, lo que contrasta con la explicación encontrada antes de la intervención, donde algunos participantes le asignaron efectos positivos y negativos, y sólo a nivel personal, sobre todo a la marihuana. Este aumento de complejidad en la teoría subjetiva permite hipotetizar una mayor disposición a la crianza preventiva.

... he aprendido a hacer cosas con la marihuana... que ni me imaginaba hacer... porque hago cosas de maderas, en huesos, hago esculturas, jamás he estudiado para eso jamás... fumo mis cuetes en mi casa y me pongo hacer cosas que ni siquiera tenía en mi mente pensado que podía hacerlas (p3, gdI).

Investigador: ¿Qué sería consumir drogas para ustedes?

Muerte... (p8, gdIII).

La idea es no consumir vicios... porque sí, al grupo familiar y a las personas, en general a toda la comunidad, afecta a todo un grupo el consumo de drogas. (p3, gdIII).

\subsection{RECONSTRUCCIÓN DE LAS TEORÍAS SUBJETIVAS SOBRE PREVENCIÓN DEL CONSUMO DE DROGAS EN LOS HIJOS(AS)}

En una etapa inicial, los padres explican que la prevención se debe aplicar cuando se presenta el problema de consumo en los hijos(as) o hay señales de riesgo, lo que se asociaría a una orientación a la acción reactiva en el proceso preventivo. El cambio de esta teoría subjetiva consiste en un desarrollo más preciso de lo que es la prevención, el otorgamiento de mayor valor y la asociación con la calidad de vida de los hijos y parientes.

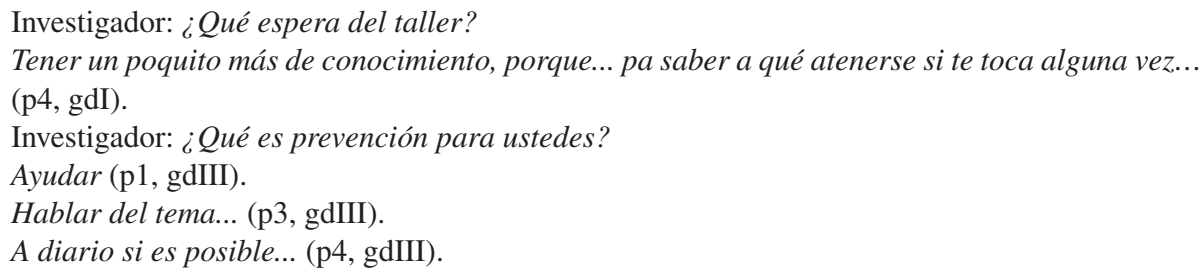

También se evidencia un cambio en la explicación de cómo se previene. Antes de la intervención, la prevención es posible, pero depende fundamentalmente de factores externos que consideran incontrolables. El cambio implica una mayor elaboración de la teoría subjetiva sobre prevención, incluyendo a la familia como el factor más importante en esta tarea y considerando que es un trabajo a realizar permanentemente, lo que se asociaría con una mayor agentividad en la prevención del consumo de drogas en los hijos(as).

La familia es el pilar número uno para educar... ahí parte todo, porque si está mal la familia está todo mal... (p2, gdIII).

Yo creo que es... el grupo familia es lo más importante para empezar a prevenir el consumo de drogas en los hijos (p1, gdIII).

\subsection{EL MOTOR DEL PROCESO DE RECONSTRUCCIÓN DE LAS TEORÍAS SUBJETIVAS}

Los participantes advierten el proceso de cambio en sus teorías subjetivas, lo consideran una transformación en su forma de vida y lo relacionan con factores propios del taller 
educativo. Así lo expresan algunos padres:

Para mí... a mí me cambió... a mí me cambió la manera de ser, yo antes no pensaba lo que pienso ahora, con este taller me ha servido bastante... (p6, gdIII).

...cambió... un gran cambio, sí como todos, está todo a la vista, ya por el hecho de entregarnos así abiertamente eso ya es un cambio inmenso ya, conocer las inquietudes que hay alrededor y que a la vuelta de la esquina y todo, sí pu... yo por mi parte quedé tiqui taca... (p2, gdIII).

Específicamente, los factores asociados al cambio representacional son:

1. Las características del educador que facilita el cambio.

Las características del educador son un factor importante en los procesos de cambio representacional. Debe transmitir a los aprendices confianza, credibilidad y competencia en lo que enseña, de lo contrario, es probable que el grupo no se disponga al cambio de las teorías subjetivas.

Porque uno puede tener muchos deseos de expresar sus cosas... que nos entendieran también a nosotros... entonces ustedes también van viendo harto de nosotros cómo somos y una convivencia así como esta que tenemos hoy en día, cuesta compañero... (p8, gdIII).

Pero será porque ellos inspiraron la confianza para generar esto... porque son profesionales... (p6, gdIII).

\section{El trabajo grupal.}

Un segundo elemento que favoreció la reconstrucción de teorías subjetivas quedó representado por la relación interpersonal grupal. Promover el autoconocimiento y análisis de las teorías subjetivas en un grupo de pares posibilitó contrastar, reflexionar y discutir distintas formas de interpretar los hechos, conllevando al desarrollo de una teoría subjetiva más colectiva y elaborada.

Haber trabajado en grupo, haber trabajado en grupo, yo me acuerdo que trabajé con el caballero M., el caballero P.... entonces yo creo que ver un, por ejemplo yo tuve mucha atención en escucharlo y creo que de sus vivencias uno aprende... (p3, gdIII).

Porque deben darse cuenta que somos todos personas... difíciles, lo más conflictivo... lo más complicado es abrirse como ustedes nos explicaban, ahí está el buen resultado de todo, apoyo de ustedes y está el apoyo de nosotros (p5, gdIII).

\section{Teorías subjetivas epistemológicas.}

Un tercer factor queda representado por la indagación y análisis de las teorías subjetivas del conocimiento, la enseñanza y el aprendizaje de la prevención del consumo de drogas. Este tipo de teorías subjetivas condiciona la disposición a aprender, por lo que abordarlas se torna crucial para el cambio representacional.

Una consulta.... una consulta... primero antes de saber todo esto.... ¿Ustedes probaron la droga?... para saber nuestra opinión... una experiencia de algo, ique si ustedes nos están preparando a las personas que fuman... ustedes tienen que tener experiencia con drogas también...! (p2, gdI).

Es que si no estaríamos basado en que todas las personas para poder educar o enseñar 
o prevenir algo tendríamos que pasar por ese proceso. No es así. No es necesario pasar por las $\operatorname{cosas}(\mathrm{p} 3, \mathrm{gdIII})$.

Investigador: ¿Cómo se puede aprender de prevención?

E... capacitándose... o planteando el tema... planteamos el tema... (p1, gdIII).

4. El autoconocimiento de las teorías subjetivas sobre consumo de drogas y su prevención.

Un cuarto factor necesario para el cambio, lo representa la indagación y análisis de las teorías subjetivas sobre el tema que se pretende enseñar. El proceso de cambio se produce cuando el participante toma conciencia de la manera en que explica la temática, cómo esta teoría subjetiva orienta la forma de abordar la crianza preventiva y de la posibilidad de reconstruir una teoría subjetiva de mayor funcionalidad.

Nosotros antes nunca nos habíamos juntado a hablar de estos temas... entonces eso ayudó... (p1, gdIII).

Para mí fue una enseñanza buena, porque ignoraba muchas cosas, que ahora sí las rescaté en realidad, ya se me quedaron grabadas en el disco duro y hay que tratar de aplicarlas lo que mejor se pueda no más, no el 100\% pero de a poquito se empieza ¿̨o no? (p2, gdIII).

\section{El uso de un lenguaje con sentido para los participantes.}

Un quinto factor que facilita el cambio es la utilización de un lenguaje que otorgue sentido a las tareas que se presentan. En esta intervención gran parte de los ejemplos y ejercicios se contextualizaron en la vida laboral de los participantes, lo que generó motivación y mayor comprensión.

\section{El insumo de conocimiento para el enriquecimiento de las teorías subjetivas.}

Un sex to factor que promueve el cambio lo representa la nueva información sobre drogas y su prevención, tarea que no sólo la realiza el educador proporcionando conocimiento científico, sino que también los participantes, con conocimiento de la vida cotidiana.

\footnotetext{
A mí me sirvió la penúltima clase, el penúltimo taller, ahí hablamos harto de lo que era familia, qué se yo, más prevención... (p1, gdIII).

Investigador: Digamos los contenidos...

Claro (p1, gdIII).

Como que por ahí fue la que más entró y porque infundir los caminos que hay que seguir, ese es el punto principal... (p2, gdIII).
}

\section{DISCUSIÓN}

Las explicaciones que los padres presentan acerca del consumo de drogas y su prevención parecen tener una relación importante con las pautas de crianza que utilizan para prevenir el consumo de drogas en sus hijos(as). Los hallazgos de este estudio permiten complementar el modelo teórico de creencias familiares del consumo de drogas (Becoña, 2001; Pons y Berjano, 1999; Secades, Fernández y Vallejo, 2005) desde una corriente más específica de investigación, esto es, las teorías subjetivas, que corresponden a un tipo de pensamiento elaborado en la vida cotidiana, que posee una estructura argumentativa y por 
lo tanto, permite explicar el propio comportamiento y los fenómenos del medio, regulando en algún nivel las acciones (Castro, 2008; Catalán, 2010; Flick, 2004).

Los hallazgos develan que estos padres tienen explicaciones generales y especificas del consumo de drogas y su prevención en los hijos(as). Las primeras, se asocian a una orientación a la acción que inhibe el proceso de cambio o aprendizaje y se organizan en un sistema supraordenado (Catalán, 2010), son de mayor generalización y actúan principalmente enjuiciando el conocimiento, enseñanza y aprendizaje en un contexto educativo, razón por la que se le ha denominado teoría subjetiva epistemológica. Éstas tendrían una fuerte implicancia en los procesos de cambio, dado que se vinculan con la disposición al aprendizaje, en este caso, sobre consumo de drogas y su prevención en los hijos(as).

Algunos autores (Hofer y Pintrich, 1997; Kember, 2001; Schommer, 1993) han encontrado un tipo particular de creencias en estudiantes universitarios, que se refieren a la naturaleza del conocimiento, sus fuentes y forma de aprenderlo, a las que denominaron epistemológicas y le asignaron un papel importante en los procesos de aprendizaje. Otros estudios de teorías subjetivas han hecho referencia a las explicaciones que los docentes tienen acerca de la enseñanza, el aprendizaje y el proceso de formación profesional (Castro, 2008; Cuadra, 2009, 2013).

En este estudio se ha visto que este tipo de teorías subjetivas se manifiestan en el participante cuando: (a) lo que se pretende es el cambio representacional; (b) este cambio implica reconstruir teorías subjetivas relacionadas con la identidad personal o algún ámbito de vida importante; y (c) cuando este proceso de cambio se realiza por medio de una instancia educativa formal. En este caso, la intervención aplicada tuvo como objetivo reconstruir teorías subjetivas relacionadas con la crianza preventiva del consumo de drogas, lo que implicó que los participantes sometieran a juicio su paternidad, rol que tiene un papel importante en la construcción de la identidad personal (Fuller, 2000; Jiménez, 2001; Viveros, 2000).

En contexto psicoterapéutico, se ha visto que la valoración que se hace de la psicoterapia y de la competencia de terapeuta (Krause et al. 2006; Krause y Dagnino, 2006) tendría una incidencia importante en la disposición al cambio subjetivo. Desde el cambio representacional (Pozo, 1999; Pozo y Gómez, 1998) también se ha planteado que este proceso se logra cuando incluye lo epistemológico y ontológico. Probablemente este tipo de teorías subjetivas se ubica en una posición de dominio (Vosniadou, 1994) o nivel supraordenado (Catalán, 2010), por lo que su identificación y abordaje se torna decisivo cuando lo que se pretende es educar a los padres en la crianza, en este caso, respecto a cómo prevenir el consumo de drogas en sus hijos(as), representando un primer paso en la intervención psicoeducativa, dado que es decisivo en la disposición al aprendizaje o al proceso de cambio. Este hallazgo podría contribuir en la comprensión del bajo nivel de eficacia que han tenido los programas de prevención del consumo de drogas, que sólo se han focalizado en la exposición de nueva información (Lloret et al. 2013; Rementería, 2001).

Las teorías subjetivas específicas de consumo de drogas y su prevención, encontradas antes de la aplicación del taller educativo, se caracterizaron por la presencia de un contenido y estructura argumentativa que limitaba en los padres y madres la acción preventiva, lo que es consistente con el estudio de Sierra et al. (2005), quien observó en éstos explicaciones insuficientes y contradictorias sobre consumo de drogas y su prevención. En los hallazgos aquí expuestos, destaca la atribución de control externa frente al consumo de drogas en 
Estudios Pedagógicos XLII, Nº 1: 283-298, 2016

CAMBIO REPRESENTACIONAL EN LOS PADRES: RECONSTRUCCIÓN DE TEORÍAS SUBJETIVAS DE LA

PREVENCIÓN DEL CONSUMO DE DROGAS EN LOS HIJOS E HIJAS

los hijos(as) y la simplicidad de las explicaciones, lo que podría asociarse a una baja agentividad y probables errores al ejercer este tipo de crianza, característica que también se ha observado en algunos estudios de teorías subjetivas en docentes (Castro, 2008; Castro y Cárcamo, 2012; Catalán, 2004; Cuadra, 2009, 2013).

Los principales cambios representacionales asociados a la intervención que realicé, consisten en una mayor elaboración de las teorías subjetivas a partir de un enriquecimiento (Carey, 1985; Moreira y Greca, 2003; Pozo, 1999) con nuevo conocimiento de tipo científico, valórico y de la vida cotidiana, lo que se puede representar en seis momentos:

(a) Autoconocimiento de las teorías subjetivas epistemológicas, que permite el reconocimiento de un metaconocimiento sobre el proceso educativo (el conocimiento que se pretende enseñar, la enseñanza y el aprendizaje de la prevención);

(b) enjuiciamiento de las teorías subjetivas epistemológicas o análisis crítico de la implicancia que tiene esta manera de pensar, en la posibilidad de cambio o aprendizaje;

(c) autoconocimiento de las teorías subjetivas específicas de drogas y su prevención, que permite reconocer las explicaciones acerca de drogas y su prevención;

(d) enjuiciamiento de las teorías subjetivas específicas de drogas y la prevención, que implica analizar la funcionalidad de esta forma de pensar;

(e) flexibilización del sistema de teorías subjetivas específicas o aceptación de la necesidad de cambio;

(f) enriquecimiento de las teorías subjetivas específicas con nueva información.

Durante todo este proceso, los elementos favorecedores del cambio son: la explicitación sucesiva de las teorías subjetivas, dado que permite un mayor grado de conciencia del propio pensamiento (Pozo, 1987, 1999, 2001, 2002) y constituye uno de los objetos de análisis cuando se pretende un aprendizaje significativo; la reflexión sobre las teorías subjetivas epistemológicas y específicas del tema, que representa el fundamento para promover el cambio, permite elaborar nuevas interpretaciones del mundo y de sí mismo (Catalán, 2011), favorece la contrastación y argumentación de modelos de conocimiento (Pozo, 1999), y que en psicoterapia es vista como una autoevaluación crítica de la propia forma de pensar (Krause et al. 2006; Krause y Dagnino, 2006); la relación interpersonal entre el educador y aprendiz, que en psicoterapia ha sido relacionado con la percepción de competencia del terapeuta (Krause et al. 2006; Krause y Dagnino, 2006) y que, desde el paradigma cualitativo aquí utilizado, incluye el respeto, la valoración del conocimiento de la vida cotidiana del participante y el abordaje del problema de estudio desde su propio marco interpretativo (Taylor y Bogdan, 1987), lo que permite una relación de confianza necesaria para este tipo de cambio; el trabajo grupal, que facilitó el abordaje de teorías subjetivas colectivas y su reconstrucción no sólo a partir del conocimiento científico propiciado por el educador, sino que también desde el propuesto por los propios participantes, más cercano, práctico y funcional $\mathrm{y}$, por ende, susceptible de aprender significativamente; el uso de un lenguaje con sentido, que permite una integración jerárquica o la relación del nuevo conocimiento con las preconcepciones (Pozo, 1987, 1999, 2002; Pozo y Gómez, 1998).

En cuanto al enfoque metodológico de este estudio, el uso de grupos de discusión como instrumento de recolección de información parece ser una opción viable cuando lo que se pretende es la identificación de teorías subjetivas colectivas o de procesos sociales de cambio (Banchs, 2000; Catalán, 2010, 2011), por su capacidad favorecedora de la discusión grupal y contrastación de interpretaciones o de modelos de conocimiento (Pozo, 
1999), a propósito del arraigo cultural (Pozo, 2002) que presentan las teorías subjetivas y que éstas se construyen en la interacción social (Catalán, 2010). En segundo lugar, parece factible aplicar mediante un grupo de discusión, la estrategia metodológica de la entrevista episódica de indagación de episodios biográficos, para facilitar la producción de teorías subjetivas (Flick, 2004), no sólo porque la narración biográfica promueve la explicitación de las mismas, sino que porque además, durante la intervención educativa permite que el cambio representacional se inicie desde las experiencias de la vida cotidiana de los participantes, otorgándole un mayor sentido al conocimiento científico que se pretende enseñar, constituyéndose finalmente este proceso en un enriquecimiento de las preconcepciones (Pozo, 2001, 2002).

Resta decir que los principales resultados que aquí se presentan podrían representar un aporte para el proceso de cambio subjetivo, específicamente, en contextos educativos, cuando lo que se pretende es desarrollar aprendizajes significativos, duraderos o en palabras de Carey (1985), un cambio fuerte, pero sólo desde una realidad local, por lo que se debe asumir a modo de hipótesis para unidades de análisis de similares características.

Diagrama 1. Modelo comprensivo del proceso de cambio representacional en los participantes

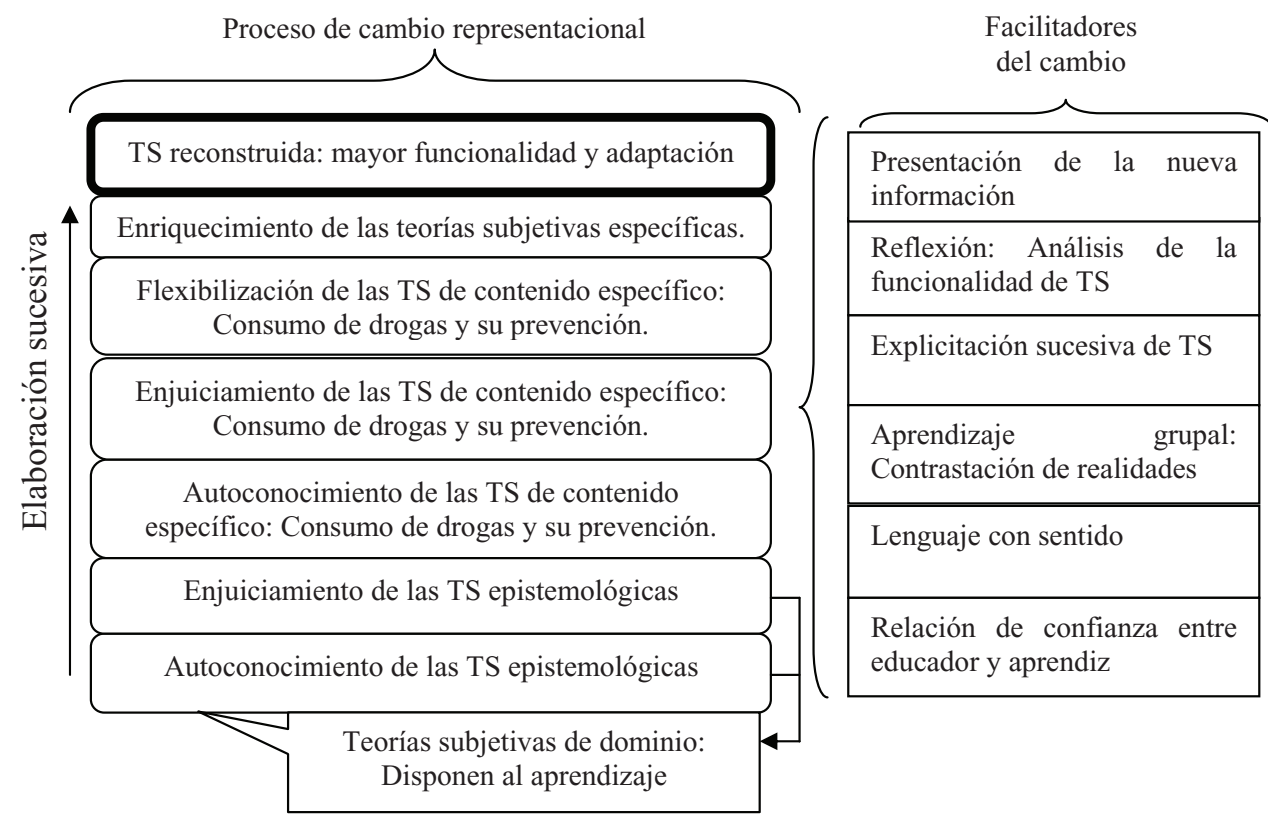

Nota: El proceso de cambio representacional se inicia de abajo hacia arriba, como lo indica la flecha del costado izquierdo. TS = Teoría subjetiva. 
Estudios Pedagógicos XLII, Nº 1: 283-298, 2016

CAMBIO REPRESENTACIONAL EN LOS PADRES: RECONSTRUCCIÓN DE TEORÍAS SUBJETIVAS DE LA

PREVENCIÓN DEL CONSUMO DE DROGAS EN LOS HIJOS E HIJAS

\section{REFERENCIAS BIBLIOGRÁFICAS}

Alfonso, J., Huedo-Medina, T. y Espada, J. (2009). Factores de riesgo predictores del patrón de consumo de drogas durante la adolescencia. Anales de Psicología, vol.25, n.2, 330-338.

Banchs, M. (2000). Aproximaciones procesuales y estructurales al estudio de las representaciones sociales. Papers on social Representations, vol.9, 3.1-3.15. [Fecha de consulta: 02 de septiembre de 2014]. Disponible en www.psych.lse.ac.uk/psr/PSR2000/9_3Banch.pdf

Becoña, E. (2001). Factores de riesgo y protección familiar para el uso de drogas. En J. Fernández y R. Secades (Eds.), Intervención familiar en la prevención de las drogodependencias (pp. 117141). Madrid: Ministerio del Interior, Plan nacional sobre las drogas.

Brotherhood, A. \& Sumnall, H. (2011). European drug prevention quality standards. A manual for prevention professionals. Luxembourg: Publications Office of the European Union. [fecha de consulta: 13 de agosto de 2013]. Disponible en http://www.emcdda.europa.eu/attachements. cfm/att_218446_EN_TD0113424ENN.pdf

Burgos, N. (2011). Investigación cualitativa. Miradas desde el Trabajo Social. Buenos Aires: Espacio editorial.

Carey, S. (1985). Conceptual change in childhood. Cambridge, MA: MIT Press/Bradford Books.

Castro, P. (2008). Cambio de teorías subjetivas de profesores respecto a la enseñanza y el aprendizaje de valores. Tesis para optar al grado de Doctor en Psicología, Facultad de ciencias sociales, Pontificia Universidad Católica de Chile, Santiago, Chile.

Castro, P. y Cárcamo, R. (2012). Cambio de teorías subjetivas de profesores respecto a la educación en valores. Revista Subjetividad y procesos subjetivos, vol.16, n.1, 17-42.

Catalán, J. (2011). Del pensamiento al conocimiento profesional del profesor. En J. Catalán (Ed.), Psicología Educacional. Proponiendo rumbos, problemáticas y aportaciones (pp. 187-215). La Serena: Editorial Universidad de La Serena.

. (2010). Teorías Subjetivas. Aspectos teóricos y prácticos. La Serena: Editorial Universidad de La Serena.

. (2004). Formación inicial de Educadoras de párvulos: un estudio de caso desde las teorías subjetivas de formadores y formadoras. Revista Iberoamericana de educación, n.33, 2-13.

Cuadra, D. (2013). Teorías subjetivas en profesores acerca de su formación profesional. Tesis para optar al grado de Magíster en Psicología, Universidad De La Serena, La Serena, Chile.

. (2009). Teorías subjetivas en docentes de una escuela de bajo rendimiento, sobre la enseñanza y el aprendizaje de alumno. Revista mexicana de investigación educativa, vol.14, n.42, 939-967.

Driver, R., Squires, A., Rushworth, P. \& Wood-Robinson, V. (1994). Making sense of secondary school. Londres: Routledge.

Elzo, J., Comas, D., Laespada, M., Salazar, L. y Vielva, I. (2000). Las culturas de las drogas en los jóvenes: Ritos y fiestas. Vitoria-Gasteiz: Servicio Central de Publicaciones del Gobierno Vasco.

Flick, U. (2004). Introducción a la investigación cualitativa. Madrid: Ediciones Morata S.L.

Fuller, N. (2000). Significados y prácticas de paternidad entre varones urbanos del Perú. En N. Fuller (Ed.), Paternidades en América Latina (pp. 35-89). Lima: Fondo Editorial de la Pontificia Universidad Católica del Perú.

Grotjahn, R. (1991). The research programme Subjective Theories. Studies in second language acquisition, vol.13, 187-214.

Hofer, B. \& Pintrich, P. (1997). The development of epistemological theories: beliefs about knowledge and knowing and their relation to learning. Rev Educ Res., vol.67, n.1, 88-140.

Jiménez, M. (2001). La reproducción de los varones en México. El entorno sexual de la misma, estudios de casos. Tesis para optar al grado de Doctor en Sociología, FCPyS, UNAM, Ciudad de México, México.

Kember, D. (2001). Beliefs about knowledge and the process of teaching and learning as a factor in adjusting to study in higher education. Stud High Educ., vol.26, n.2, 205-221. 
Krause, M., De La Parra, G., Arístegui, R., Dagnino, P., Tómicic, A., Valdés, N., Vilches, O., Echávarri, O., Ben-Dov, P., Reyes, L., Altimir, C. y Ramírez, I. (2006). Indicadores genéricos de cambio en el proceso psicoterapéutico. Revista latinoamericana de psicología, vol.38, n.2, 299-325.

Krause, M. y Dagnino, P. (2006). Evolución del cambio en el proceso psicoterapéutico. Gaceta Universitaria, vol.2, n.3, 287-298.

Larriba, J., Duran, A. y Suelves, J. (2006). Programa de prevención familiar del consumo de drogas. Barcelona: Editorial PDS Promoción y desarrollo social.

Lloret, D., Espada, J., Cabrera, V. y Burkhart, G. (2013). Prevención familiar del consumo de drogas en Europa: una revisión crítica de los programas contenidos en EDDRA. Adicciones, vol.25, n.3, 226-234.

Marín, N. (1999). Delimitando el campo de aplicación del cambio conceptual. Enseñanza de las ciencias, vol.17, n.1, 80-92.

Martínez, A. (2001). Familia y consumo de drogas desde el Modelo Circumplejo de evaluación familiar. En I. Vielva, L. Pantoja y J. Abeijón (eds.), Las familias y sus adolescentes ante las drogas. El funcionamiento de la familia con hijos de comportamiento no problemático, consumidores y no consumidores de drogas. Avances en drogodependencias (pp. 51-100). Bilbao: Universidad de Deusto.

Moreira, M. y Greca, I. (2003). Cambio conceptual: análisis crítico y propuestas a la luz de la teoría de aprendizaje significativo. Ciência \& Educação, vol.9, n.2, 301-315.

Navarro, J. (2001). Los andaluces ante las drogas VII. Sevilla: Junta de Andalucía. Consejería de Asuntos Sociales. Comisionado para las drogodependencias.

Nuño, B. y González, C. (2004). La representación social que orienta las decisiones paternas al afrentar el consumo de drogas en sus hijos. Salud Pública de México, vol.46, n.2, 123-131.

Oliva, J. (1999). Algunas reflexiones sobre las concepciones alternativas y el cambio conceptual. Enseñanza de las Ciencias, vol.17, n.1, 93-107.

Pons, D. y Berjano, P. (1999). El consumo abusivo de alcohol en la adolescencia. Un modelo explicativo desde la psicología social. Madrid: Servicio de Publicaciones del Plan Nacional sobre Drogas. [Fecha de consulta: 24 de septiembre de 2013]. Disponible en http://www.pnsd. msc.es/Categoria2/publica/pdf/cabu.pdf

Pozo, J. (2002). La adquisición de conocimiento científico como un proceso de cambio representacional. Investigações em Ensino de Ciências, vol.7, n.3, 245-270.

. (2001) Humana mente: el mundo, la conciencia y la carne. Madrid: Morata.

(1999). Más allá del cambio conceptual: El aprendizaje de la ciencia como cambio representacional. Debates, vol.17, n.3, 513-520.

. (1987). Del pensamiento formal a las concepciones espontáneas: ¿Qué cambia en la enseñanza de la ciencia?. Infancia y aprendizaje, n.38, 35-52.

Pozo, J. y Gómez, M. (1998). Aprender y enseñar ciencia. Madrid: Morata

Rementería, I. (2001). Prevenir en drogas: paradigmas, conceptos y criterios de intervención. Santiago: Publicación de las Naciones Unidas.

Rodrigo, M., Rodríguez, A. y Marrero, J. (1993). Las teorías implícitas: una aproximación al conocimiento cotidiano. Madrid: Visor.

Saez, X. (2002). Análisis de efectividad de campañas masivas de prevención de drogas. Santiago: Fundación Paz Ciudadana. [fecha de consulta: 22 de marzo de 2014]. Disponible en http://www. pazciudadana.cl/publicacion/analisis-de-efectividad-de-campanas-masivas-de-prevencion-dedrogas/

Schommer, M. (1993). Comparisons of beliefs about the nature of knowledge and learning among postsecondary students. Res High Educ., vol.34, n.3, 355-370.

Secades, R. (1996). Alcoholismo juvenil. Prevención y tratamiento. Madrid: Pirámide.

Secades, R., Fernández, J. \& Vallejo, G. (2005). Family risk factors for adolescent drug misuse in Spain. Journal of Child \& Adolescent Substance Abuse, vol.14, 1-15. 
Estudios Pedagógicos XLII, N $1:$ 283-298, 2016

CAMBIO REPRESENTACIONAL EN LOS PADRES: RECONSTRUCCIÓN DE TEORÍAS SUBJETIVAS DE LA

PREVENCIÓN DEL CONSUMO DE DROGAS EN LOS HIJOS E HIJAS

Sierra, D., Pérez, M., Pérez, A. y Núñez, M. (2005). Representaciones sociales en jóvenes consumidores y no consumidores de sustancias psicoactivas. Adicciones, vol.17, n.4, 349-360.

Silveira, F. (1991). A filosofia da ciência de Karl Popper e suas implicações no ensino da ciência. En M. Moreira y R. Axt (Eds.), Tópicos em ensino de ciências (pp. 62-78). Porto Alegre: Sagra.

Strauss, A. \& Corbin, J. (2002). Bases de la investigación cualitativa. Técnicas y procedimientos para desarrollar la teoría fundamentada. Antioquia: Editorial Universidad de Antioquia. Primera Edición en Español.

Taylor, S. y Bogdan, R. (1987). Introducción a los métodos cualitativos de investigación. Barcelona: Paidós.

Tsukame, A. (2002). El consumo de drogas en busca de sentido. En M. Hopenhayn (Ed.), Prevenir en drogas: enfoques integrales y contextos culturales para alimentar buenas prácticas (pp. 29-38). Santiago: Naciones Unidas.

Vielva, I. (2001). La disciplina y las prácticas educativas. En I. Vielva, L. Pantoja, J. Abeijón (Eds.), Las familias y sus adolescentes ante las drogas. El funcionamiento de la familia con hijos de comportamiento no problemáticos, consumidores y no consumidores de drogas. Avances en drogodependencias (pp. 101-150). Bilbao: Universidad de Deusto.

Viveros, M. (2000). Paternidades y masculinidades en el contexto colombiano contemporáneo, perspectivas teóricas y analíticas. En N. Fuller (Ed.), Paternidades en América Latina (pp. 91127). Lima: Fondo Editorial, Pontificia Universidad Católica del Perú.

Vosniadou, S. (1994). Capturing and modelling the process of conceptual change. Learning and Instruction, vol.4, n.1, 45-69. 